\title{
REVISIÓN DE ALGUNAS PROPUESTAS TEÓRICAS EN TORNO A LA CARACTERIZACIÓN DEL MODO SUBJUNTIVO
}

\author{
Jorge Murillo Medrano
}

\begin{abstract}
RESUMEN
En este trabajo se revisan, de manera crítica, las concepciones básicas sobre el modo subjuntivo y la modalidad dentro de la tradición de estudios hispánicos, tratando de dar un panorama completo y contrastivo.
\end{abstract}

\section{$\operatorname{ADSTRACT}$}

This article focuses especifically on the different conceptions about the subjuntive mood and the concept of modality in the traditions of Hispanic studies. It pretends to give a whole panoram contrasting the different positions in a critical way.

Para dar una visión de conjunto sobre las concepciones del modo subjuntivo se impone, en primer lugar, la revisión de los conceptos de la gramática normativa tradicional, los cuales están bien representados por Andrés Bello y por la Real Academia Española, continuadora y legitimadora de toda una tradición de estudios lingüísticos iniciados ya desde Nebrija, y cuyo marco fundamental son las ideas gramaticales de la antigua latinidad.

Para Bello (1988: 363), los modos son "las inflexiones del verbo en cuanto provienen de la influencia o régimen de una palabra o frase a que esté o pueda estar subordinado". Aclara, en seguida, que el término regente puede estar implícito, como idea dominante que eventualmente pudiera representarse como una oración principal.

En cuanto al subjuntivo, Bello distingue varios tipos: (¿enumeración?) el subjuntivo común, que abarca aquellos casos que pueden subordinarse a verbos de duda o deseo y el optativo, que comprendería “...las subjuntivas comunes que se emplean en proposiciones independientes para significar el deseo de un hecho positivo o negativo..." (363). [Como una variación de esta última categoría incluye todas las formas del imperativo en tanto expresan deseos que debe ejecutar el interlocutor.] El segundo gran tipo de agrupación correspondería al subjuntivo hipotético, es decir, aquellos casos en que se enuncia condición o hipótesis. [Hace la observación de que este modo es peculiar de la conjugación castellana, ya que no existió en latín y 
tampoco se da en las otras lenguas romances. Los tiempos verbales de este grupo serían ambos futuros de subjuntivo (el simple y el compuesto), aunque hace la observación de que este último se confunde con las formas en - se y -ra del subjuntivo común.]

La importancia de la aportación de Bello la resume muy bien Navas Ruiz (1990) al afirmar que "Bello aporta varias nociones importantes a la teoría de los modos: a) sostiene que tanto el indicativo como el subjuntivo y el optativo son modos subordinados o pueden serlo; b) que en toda proposición ha de entenderse siempre un subordinante, explícito o implícito; c) que en la elección del modo se combinan criterios sintácticos (-palabras regentes-) con semánticos (verbos de afirmación, de duda)" (112).

En la última gramática publicada por la Real Academia, el Esbozo para una Nueva Gramática de la Lengua Española (1973), se define el "modo verbal" en los siguientes términos: "En varias ocasiones hemos distinguido el contenido de lo que se dice (dictum) de cómo lo presentamos en relación con nuestra actitud psíquica (modus). Entre los medios gramaticales que denotan la actitud del hablante respecto a lo que se dice, se encuentran las formas de la conjugación conocidas por antonomasia con el nombre tradicional de modos" (454).

Seguidamente, se establecen cuáles son los modos dentro de la lengua española, con su respectiva caracterización linguiística: el indicativo, el subjuntivo y el imperativo. El primero como expresión de la realidad y como miembro no marcado o indiferenciado de la expresión verbal; el segundo, como expresión de la no realidad y, por ende, como elemento marcado de la expresión; en cuanto al último, el imperativo, se especifica que "...responde exclusivamente a la función áctica del lenguaje, y expresa exhortación, mandato o ruego dirigidos a otra persona, de la cual depende que la acción se realice o no" (454).

Para la caracterización particular del modo subjuntivo, se parte de la significación misma de la palabra, "subjuntivo" como "subordinado", y por ende se establece que este sería el modo de subordinación por excelencia (-aunque no necesariamente exclusivo-). Se señala, por otra parte, aunque no se aclara, el hecho de que el subjuntivo aparece en variadas oraciones simples e independientes también.

El criterio organizador que utiliza la Academia para los tiempos del modo subjuntivo sigue la tradición latina de división en subjuntivo común o dubitativo y el optativo: "La distinción es útil para el español, porque establece con claridad dos tipos bien diferenciados de subjuntivo, a los cuales llamaremos respectivamente potencial y optativo. Comprende el primero las acciones pensadas como dudosas o posibles, y el segundo las necesarias o deseadas" (455).

Siguiendo estos lineamientos, la Academia organiza la presentación de los tiempos del modo subjuntivo en dos grandes apartes, que corresponden a las dos categorías mencionadas. A su vez, la caracterización responde al criterio sintáctico de aparición del verbo en oraciones subordinadas o bien en frases independientes. Al criterio sintáctico anterior, se une además el semántico, en tanto se hace referencia siempre, en el caso de la subordinación, al tipo de verbo subordinante (de duda, de emoción, de necesidad subjetiva, por ejemplo) para justificar la elección del modo subjuntivo.

Las carencias de la caracterización anterior son evidentes: en primer lugar, la correspondencia subjuntivo=irrealidad no podría sostenerse en frases del tipo:

(1) Me duele que te hayan robado tu dinero 
en la que la acción expresada en subjuntivo no podría ser más real que cualquiera expresada en indicativo. Al respecto, Brian Castranovo (1990) apunta que: "Para justificar el uso del subjuntivo en esos casos, se sigue el curso algo especioso de aceptar que "es tal la fuerza subjetivadora de los sentimientos, que imprimen su matiz modal al verbo subordinado, afirme o no la realidad del hecho" ( 9 3 3.13.3.2d) ( 79 ).

Por otra parte, el binarismo (propio de la gramática estructural) en que se fundamenta la separación indicativo/subjuntivo no da cuenta exacta de la posición del imperativo, sobre todo tomando en cuenta que subjuntivo e imperativo comparten, en buena medida, formas verbales. Los límites entre estos dos últimos se establecen echando mano a otro criterio, el pragmático, pues se aduce que el imperativo corresponde a la dimensión apelativa del lenguaje.

Los límites indicativo/subjuntivo quedan también inseguros, sobre todo cuando se trata de frases en las que pueden aparecer ambos o en las que se utiliza una forma del condicional (indicativo) con contenido semántico de irrealidad o inseguridad por parte del hablante, como en:

$$
\text { Tal vez llegaría ya. }
$$

La misma clasificación entre optativo y dubitativo no podría explicar aquellos casos de oraciones subordinadas de sustantivo o adjetivo, como en:

(3) El hecho de que llegara tarde, me molestó mucho.

(4) No lo digo en el sentido de que tenga que ser así.

El problema de la aparición del modo subjuntivo en oraciones independientes, lo cual contradiría el mismo étimo del vocablo "subjuntivo", se justifica en tanto que, en esos casos, "...el subjuntivo señala el carácter volitivo, dubitativo o afectivo, ayudándose de partículas o del sentido y, en la lengua hablada, de la entonación, como en Dios le asista; Sea enhorabuena;..." (Real Academia, 455).

El anterior.intento de explicación por parte de la Academia, queda mejor dilucidado por el gramático Samuel Gili Gaya, quien en su Curso Superior de Sintaxis Española (1993) da una explicación que concierta mejor con el concepto de subjuntivo como modo de subordinación: "Se trata (en esos casos) de subordinaciones mentales que envuelven psíquicamente al juicio que se enuncia, aunque gramaticalmente no dependa de un verbo principal" (132). De esta manera queda normalizado el panorama, al asumir que en todos los casos el subjuntivo aparecería, explícita o tácitamente, dentro de una estructura subordinada.

Lo anterior nos da posibilidad de pasar revisión de las conceptualizaciones de Gili Gaya en relación con el subjuntivo, las cuales no difieren mucho de las academicistas en tanto que, según Ignacio Bosque (1990), "como este autor contribuyó decisivamente a la redacción del Esbozo académico, es lógico que también esta obra esté en buena parte impregnada de ella (se refiere a la revisión de Gili Gaya)" (1). Sin embargo, para clasificar los diferentes usos del subjuntivo y sus entornos de aparición, este gramático parte de criterios semánticos y sintácticos que dan como resultado el mismo esquema de la Academia, a saber, subjuntivos potencial y optativo tanto en estructuras subordinadas como dependientes. 
Consciente, quizá, de que esta taxonomía no permitiría dar cuenta exacta de todos los usos del subjuntivo en la lengua, hace la aclaración de que "en el uso moderno del subjuntivo español intervienen factores psicológicos, históricos y estilísticos que vamos a tratar de valorar, no con el fin imposible de dar a los extranjeros una fórmula sencilla que prevea siempre el modo que tienen que usar, sino con el de hallar un criterio de interpretación suficientemente claro para todos los casos que encuentren en la conversación y en los textos" (133).

Por otra parte, en su fundamentación teórica retoma algunos criterios de Rodolfo Lenz (La oración y sus partes, 1920), quien partía de principios de la lógica (como se verá más adelante) para refutarlos y plantear como asidero teórico lineamientos de índole psicológica.

Instaurando otra línea de posible análisis, Lenz -en la obra mencionada- establecía un marco lógico semántico para la descripción de los modos. Define el modo como la categoría gramatical según la cual se clasifican las formas verbales, subjetivamente y desde el punto de vista del hablante, en correspondencia con su valor lógico. Para él, la diferencia entre los tres modos (indicativo, subjuntivo e imperativo) responde a distinciones lógicas entre juicios asertorios (hechos reales), juicios problemáticos (hechos dudosos o meramente posibles) y juicios apodícticos (hechos deseables o necesarios), lo cual correspondería, respectivamente, al indicativo, al subjuntivo dubitativo y al optativo.

Se le ha señalado a Lenz el hecho de que su clasificación no tiene asideros propiamente linguísticos (Castronovo, 1990); además de que, al igual que en las clasificaciones tradicionales, su visión no toma en cuenta casos en los que se usa el indicativo para expresar duda o posibilidad (Tendría que haber sido así) o, por el contrario, el subjuntivo en oraciones que aluden a hechos reales (Me duele que te hayas caído). Su perspectiva logicista, sin embargo, es muy importante en tanto vislumbra, dentro del panorama gramatical de la época, criterios que fundamentarán la gramática generativo-transformacional de la segunda mitad del siglo, los cuales serán retomados como base para una nueva propuesta por Terrel y Hooper (1974), por ejemplo, o por análisis de corte pragmático.

Otro enfoque para el análisis del subjuntivo es el presentado por Mauricio Molho (1975), quien aplica al español las ideas desarrolladas por Gustave Guillaume (1929), con Pottier (1970) y Lamíquez (1982) como algunos de sus discípulos. Según esta corriente, el modo sería una representación del tiempo dentro del sistema verbal. La imagen del tiempo, o cronogénesis, daría lugar a un continuum que estaría representado por diferentes etapas o cronotesis. Estos cortes definen el modo:

\footnotetext{
"A cada una de las intercepciones sucesivas de las que es objeto la construcción mental de la imagen-tiempo corresponde un modo del verbo, cuya representación se obtiene por medio de un corte practicado a través de la cronogénesis, la cronotesis, de modo que cada cronotesis descubre al pensamiento, que así conoce lo que en él ocurre, el resultado obtenido en cada una de las etapas sucesivas por la operación de la cronogénesis" (74).
}

Las cronotesis tienen un orden dentro de esa imagen-tiempo, es decir, su secuencia da como resultado una asignación de desarrollo de la imagen-tiempo a cada uno de sus modos; así, a la primera cronotesis corresponde el modo casi nominal, "un tiempo mínimamente construido, aunque en potencia de ser ulteriormente construido" (73). La imagen del tiempo aquí es rudimentaria, apenas alejada de lo nominal y expresada por las formas del infinitivo y gerundio para el presente y del participio para el pasado; en fin, un tiempo "in posse". 
La segunda cronotesis refiere a un tiempo imperfectamente construido, en trance de construirse, es decir, "in fieri” y se asocia con el subjuntivo. Los tiempos de este modo, más que estructuras acabadas, son tendencias, direcciones hacia lo futuro o el pasado.

En una última etapa se encuentra el indicativo, que se correlaciona con la tercera cronotesis o tiempo "in esse", es decir, tiempo acabado que se ordena desde un presente y se proyecta hacia un futuro o hacia el pasado.

Una vez descritas las tres fases de la cronogénesis, Molho aclara que estas corresponden a los modos lingüísticos y no a la modalidad, "puesto que un solo modo de la lengua se presta a la expresión discursiva de modalidades múltiples y a veces contradictorias, y que inversamente una modalidad resulta susceptible de expresarse por medio de dos, y aun tres, modalidades" (75). Por ello, no se considera al imperativo como un modo, sino como un efecto discursivo de los modos cronogenéticos al que recurre el hablante para expresar un mandato o súplica.

La distinción entre modo y modalidad es de suma importancia, pues le permite a este lingüista establecer una mejor relación de las formas del imperativo con los otros modos lingüísticos. En vista de su capacidad apelativa, el imperativo siempre ha sido tratado en forma aparte, aun a pesar de que en la mayoría de los casos comparte morfemas con el modo subjuntivo. Lamentablemente, Molho no profundiza en las bases teóricas que permitirían establecer estos lazos del imperativo con los otros modos y en los problemas de la modalidad discursiva, lo cual le lleva a ver el imperativo en forma tangencial.

Las ideas de Guillaume, base de la teoría que Molho plantea, son importantes en tanto abren nuevas posibilidades de visualizar las relaciones entre los modos y tiempos verbales, tal y como lo comprueba el hecho de que son retomadas también por Vidal Lamíquez (1972) quien las matiza con criterios estructurales. Dentro de esta misma línea funcional, se pueden situar los estudios de Alarcos Llorach (1994 y 1995), quien partiendo de criterios meramente binaristas trata de explicar el sistema verbal del español. Por ejemplo, para él, la distinción entre indicativo/subjuntivo se asienta en el principio de que el subjuntivo es el miembro marcado, es decir, el conjunto de formas verbales que "envuelven la acción en un tinte subjetivo" (1994: 62). Interesante resulta su concepción de que tanto sintáctica como morfológicamente el imperativo queda fuera del sistema, tal y como él lo concibe, puesto que sólo admite los pronombres en posición proclítica y tiene formantes morfológicos diferentes a los demás tiempos verbales. De hecho se refiere aquí a las formas propiamente del imperativo, pues evade hacer referencia a todos aquellos dialectos de la lengua en los que se toman formas del subjuntivo para expresar mandato.

Por otra parte, concibe el modo como una categoría de morfemas que presenta dirección heteronexual, es decir, que "la presencia de morfemas de indicativo o de subjuntivo depende de una noción expresada en otro nexo (=frase), al que determina" (62). Para él, entcnces, el subjuntivo estaría regido por ciertos verbos o palabras que indican ideas irreales y que pertenecerían a la frase regente.

Hasta aquí, se ha dado una visión, bastante somera, de los que consideramos los puntos clave en el desarrollo de las ideas en torno a la concepción modal dentro de la linguística hispánica. Habiendo hecho una revisión exhaustiva, se pudo notar que en la mayoría de manuales gramaticales se siguen las concepciones anteriormente esbozadas, a veces con pequeñas variantes o matices diferentes. 
La otra gran corriente que viene a tratar de explicar el subjuntivo español desde otro marco de referencia la constituye la linguística norteamericana, con acercamientos que podríamos dividir en tres grandes tipos: los que siguen criterios de la gramática tradicional (mezcla de principios semánticos y sintácticos), aquellas que se fundamentan en la gramática generativa y, en las últimas décadas, los enfoques pragmáticos. Todos estos intentos están basados en un presupuesto común: tratar de explicar el subjuntivo con propósitos pedagógicos, es decir, con la finalidad de establecer mejores taxonomías que faciliten la enseñanza del español como segunda lengua. Es de notar también el hecho de que, dada la enorme influencia de los lingüistas estadounidenses, este tipo de análisis se ha extendido al mundo hispano y actualmente los estudios de unos y otros no parecen diferir en gran medida en cuanto a lo teórico se refiere ${ }^{1}$.

Dentro del primer grupo señalado arriba, -los que se guían por principios tradicionales-, podemos citar a Robert Spaulding (1931), quien no va más allá de definir el subjuntivo como el modo de la "duda o vacilación" y de describir los diferentes usos de este modo a la manera tradicional. Para Keniston (1937) el subjuntivo es el modo de la subjetividad, en oposición a la objetividad expresada por el indicativo, y además, "it serves merely to reflect the attitude of the speaker toward a given action or state.” Sí establece que esta actitud subjetiva puede expresarse tanto en cláusulas subordinadas como en las independientes y, a la vez, divide los casos en aquellos que manifiesten deseo y los que reflejan incertidumbre. Keniston señala que el subjuntivo puede usarse como imperativo tanto en la forma negativa de segunda persona, como con el pronombre ustedes y que sintácticamente estos usos pueden aparecer sin palabras introductorias o también introducidos por que.

Después de estos dos lingüistas de la primera mitad de siglo, el interés por el subjuntivo reaparece con los estudios generativos posteriores y ahí las opiniones empiezan a proliferar y dividirse. Tomaremos las que consideramos más representativas para esbozar lo que, desde este marco gramatical, se ha planteado.

Debe aclararse, en primer lugar, que el intento por describir el funcionamiento del subjuntivo español partiendo solamente de principios sintácticos parece no haber dado resultados completamente satisfactorios y esa es la posición de Hadlich (1982) quien aboga más bien por incorporar principios semánticos: "Una descripción más precisa del uso del subjuntivo español iría así, probablemente, vinculada a una descripción de alto nivel de la semántica de las locuciones completas, en vez de a los verbos solos" (300). Así, la clasificación que este autor hace de las oraciones donde aparece el subjuntivo, se fundamenta más que todo en criterios relacionados con el significado de las frases que introducen las apariciones de estas formas verbales.

Probablemente, Terrel y Hooper ya habían percibido lo anterior y en 1974 publicaron una teoría que partía de principios semánticos (corroborados con lo sintáctico). Ellos retomaron la taxonomía original de Lenz y la desarrollaron con mucha más acuciosidad y cuidando que respondiera a principios más asociados con la linguiística que con la lógica. Fundamentalmente, plantean que "...en las oraciones independientes y en las sustantivas, el indicativo se relaciona con la aserción, mientras que el subjuntivo se asocia con la no -aserción" (150). La importancia de esta explicación radica en que se liga la visión de Lenz con los enfoques generativos y se plantea abiertamente la necesidad de partir de criterios semánticos para la explicación del subjuntivo, tarea a la que habían rehuido otros de sus colegas pues este campo, 
el de la semántica, no había sido el fuerte del generativismo. A pesar de su marcada intención de enmarcarse dentro de lo semántico, la segunda parte de su estudio da cuenta de las características sintácticas que corroboran sus ideas respecto del uso del subjuntivo.

Por lo anterior, algunos otros prefieren abordar la cuestión desde un marco psicológico, apoyados en la semántica generativa, dado que, como afirma Goldin (1974 ): “...generative semantics tries to show that the principles native speakers use to form sentences can not be stated without reference to such nonsyntactic factors as Beardsley wrote of: the nature of the situations being talked about and the speaker's beliefs" (295). Situándose en este marco, Goldin plantea que los principios que gobiernan la elección del subjuntivo no van más allá de dos o tres: cuando el hablante desea expresar una reacción ante algo que "He may like it, dislike it, be surprised by it, made sick by it, be frightened by it, appalled by it, delighted with it, happy about it, sad about, angry about it" (296) y que por lo tanto, si en el verbo subordinante se expresa alguna de las reacciones anteriores, el subordinado irá en subjuntivo. El segundo principio establece que si el hablante tiene una presuposición afirmativa sobre un hecho, este se expresará en indicativo, en tanto que si su presuposición es negativa o indefinida, usará el modo subjuntivo. Partiendo de estos dos grandes criterios, genera todos los usos del subjuntivo, haciendo la salvedad de que en las construcciones condicionales no operan estos principios de la misma forma, como también en aquellos casos en que el subjuntivo expresa reacción o presuposición, negativa o indefinida, estando en la cláusula principal.

La proliferación de estudios sobre el subjuntivo desde la gramática generativa y el interés por reducir, en lo máximo posible, todos los diferentes usos a una sola regla que facilitara su aprendizaje, lleva a Bergen (1978) a afirmar que todos los posibles usos de este modo se pueden reducir a un solo principio: "The single criterion for the use of mode is simply that, whereas the indicative denotes that the speaker (or the actor) of the higher clause regards the preposition expressed by the next lower clause as an objective fact, the subjunctive expresses a subjective reservation on the part of the speaker (or actor) concerning the reality of that preposition" (22). Bergen llega a esta conclusión impulsado por lo que él considera un exceso de reglas al respecto por parte de los estructuralistas (da cuenta de 34 reglas para el uso de este modo), las cuales, más que normalizar el panorama, no hacen sino escindirlo.

Hemos tomado la posición de Bergen porque creemos que resulta representativa de esa eclosión de teorías sobre el uso del subjuntivo en búsqueda por la supuesta fórmula que diera cuenta exacta del funcionamiento de este modo. El mismo Bergen no escapa a estas intenciones pues supedita la variación y la flexibilidad de la lengua a principios lingüístico-didácticos que a la postre imponen una visión simplista del asunto.

Lo anterior ha sido bien vislumbrado por Bell (1990), quien va en contra del establecimiento de un principio unitario y vuelve a poner el asunto en discusión. Después de pasar revista a algunas de las teorías, concluye no aceptando explicaciones unitarias o aquellas en las que se etiquetan diferentes formas bajo un mismo nombre, pues considera que en muchos casos las diferencias semánticas son mayores que sus semejanzas. Termina diciendo que prefiere "...entender el modo como parte del proceso semántico de la significación, proceso total y enormemente flexible, es decir, "lo que el hablante quiere decir"” (104).

Los enfoques pragmáticos, por su parte, se enmarcan dentro de los lineamientos sociolingüísticos y su objetivo primordial es el análisis del enunciado linguiístico en relación con los sujetos participantes en el acto comunicativo. Klein (1994) se propone hacer una ampliación de 
lo que Terrel y Hooper (1974) plantean, orientándose sobre todo a aclarar aquellos casos ambiguos, en los que cabría, dependiendo de la intención del hablante, el indicativo o el subjuntivo, como en la oración Insisto que estudie/estudia. Para ella, la competencia del hablante, por sí misma, no basta para dilucidar de qué significado se trata y sola la actuación, visualizada desde un contexto comunicativo, permitiría resolver la ambigüedad.

Otro autor que partiendo siempre de las teorías propuestas por Terrel y Hooper, las extiende echando mano del mismo enfoque pragmático es Guitart (1990: 321). Sus intenciones las resume así: "(...) explicaré la distinción "aserción"/"presuposición”, tanto en relación con la intención del hablante al formular una proposición, como en relación con lo que el oyente conoce. De modo aproximado, si el hablante transmite el complemento como información, lo está afirmando; si da una opinión acerca de éste, lo está presuponiendo. Terrel y Hooper estarían de acuerdo con esto. Lo que no tienen en cuenta es que la presuposición semántica es independiente de la afirmación".

Debe hacerse la salvedad de que todos estos estudios instaurados a partir de la posición de Terrel y Hooper sólo van dirigidos a explicar el funcionamiento del modo subjuntivo en las oraciones subordinadas sustantivas y, en menor grado, las adjetivas. Además, como en la mayoría de los análisis generativistas estadounidenses, su interés final es hacer un aporte con repercusiones didácticas, aunque al final revelen, como Terrel y Hooper, por ejemplo, que:

“...en realidad, esta propuesta, más que sustituirlo, continúa el enfoque tradicional de la enseñanza del subjuntivo español”.

La cita anterior de Terrel y Hooper es muy reveladora puesto que nos induce a preguntarnos en qué medida todos estos enfoques, que se plantean desde diferentes marcos y pretenden por ende solucionar los problemas del uso del subjuntivo, no son sino diferentes modalidades de decir lo que, desde hace muchos años, ya se ha afirmado.

Sin duda alguna, cada uno de ellos aporta, desde su ángulo, nuevas aristas del modo subjuntivo pero en principio la cuestión medular sigue siendo la misma: indicativo y subjuntivo se oponen en virtud de que hay principios semánticos y sintácticos que lo permiten, amén de la situación pragmática del habla, que en términos de la gramática tradicional no vendría a ser más que la ya trillada "intención del hablante". De este modo, las definiciones del subjuntivo como el modo de la incertidumbre, de la duda, de la posibilidad, o como decía Lenz, de los juicios apodícticos o problemáticos vendrían a ser sinónimas de las más modernas, que lo ven como el modo para expresar reserva, no-aserción o no-afirmación. Lo anterior solamente desde un marco semántico pues desde lo sintáctico, la misma restricción intrínseca del șubjuntivo de aparecer, explícita o implícitamente, en construcciones subordinadas no puede sino supeditar todos los estudios al respecto a ese rasgo.

Por otra parte, debe reconocerse, a los estudios tradicionales, ciertas ventajas sobre los enfoques modernos. En primer lugar, fundamentan su descripción en una visión de conjunto, esto es, tratando de caracterizar el modo subjuntivo como un sistema, una totalidad. Acorde con lo anterior, sus descripciones intentan dar cuenta de todos los posibles usos de este modo y no escatiman el recurrir a criterios híbridos (semánticos, sintácticos, estilísticos o incluso históricos) para darnos una visión del funcionamiento global del modo. En segundo lugar, su marcada tendencia descriptivista (y prescriptivista también) no restringe el que se mencionen 
diversas instancias de uso de estas formas verbales, sea para ratificarlas o para censurarlas y ello, a la vez, les permite mostrar las posibles fisuras del sistema.

En cuanto a los enfoques desde una perspectiva definida, que son los más usuales en los últimos años, su marcada tendencia a apegarse a un marco metodológico o descriptivo los restringe a proponer reglas o fórmulas que sólo dan cuenta de un número muy reducido de casos, normalmente limitados por rasgos sintácticos, a saber, en oraciones sustantivas, en las adjetivas o en construcciones independientes. Esta limitación del campo de análisis no ha provocado sino discusiones en torno a si esas reglas que se formulan son o no válidas como generalizaciones o si se podrían extender a otros casos. Por otra parte, el evidente interés pedagógico de los lingüistas estadounidenses los ha conducido, como hemos señalado, a caer en la trampa de supeditar todas sus observaciones y proposiciones al único fin de facilitar la enseñanza-aprendizaje de estas formas verbales en las clases de español como lengua extranjera.

Sería injusto, por otra parte, sólo tener en cuenta los aspectos negativos pues la especificidad de estos intentos ha arrojado muchísima luz sobre aspectos que la gramática tradicional, por sus limitaciones o por sus circunstancias, no había podido dilucidar. De hecho, esa especificidad permitiría hacer uso de observaciones valiosas en cuanto al funcionamiento del modo en estudios específicos que se pudieran hacer.

Las semejanzas entre las distintas posiciones esbozadas tiene una explicación lógica si se considera que el modo, como categoría gramatical, no puede escaparse de ser definido en términos de las relaciones semánticas que se establecen entre el hablante y su enunciado y, a la vez, de las interrelaciones que se ejercen entre las diferentes instancias sintácticas, sean estas sintagmas verbales, nominales o adverbiales. No podríamos vaciar el lenguaje de su contenido semántico y verlo sólo como significante, pero tampoco podríamos pensar que las estructuras de significado no están sujetas a lo sintáctico y más aún olvidar que el lenguaje es un instrumento social y que, por consiguiente, el contexto comunicativo es decisivo para la fijación del sentido. Ahí es donde, creemos, fallan aquellos enfoques que pretendan dar una explicación unilateral del subjuntivo y esto causa también que perduren, como se puede corroborar, descripciones como la de Gili Gaya, quien no escatima el hacer uso de cualquier marco siempre y cuando este le permita aclarar más el panorama.

Considerado a la luz de lo anterior, todo análisis que se intente hacer del modo subjuntivo se fundamentaría, en última instancia, dentro de esas coordenadas del sistema, si bien es cierto que podría optarse por escoger uno u otro ángulo para desentrañar las complejas relaciones que se dan en la lengua, y en ese sentido, concordamos plenamente con la propuesta de Navas (1990): "Para el análisis y la práctica conviene establecer la división tradicional en subjuntivo independiente y dependiente. Dentro de este, la división en tipos de proposiciones. Este es el único camino para una descripción metódica y exhaustiva” (139).

El otro aspecto que necesariamente debe ser clarificado, al menos para nuestros propósitos, es la concepción de "modo" sobre la cual se debe trabajar. Generalmente, los investigadores rehúyen dar una definición estipulativa de conceptos que se consideran problemáticos o difíciles de concretar y prefieren optar por la vía de la descripción o el análisis como mecanismo definitorio.

En la mayoría de los casos, se describe el modo ya sea partiendo de fundamentos psicológicos (externamente, es decir, caracterizándolo como la actitud que el hablante toma en relación con el enunciado) o desde un ángulo meramente gramatical (como un conjunto de 
morfemas que afecta a ciertas formas verbales tanto en su funcionamiento sintáctico como semántico). Los avances hechos por investigaciones recientes tratan de deslindar estos dos aspectos y de inscribirlos en marcos metodológicos específicos que permitan precisar mejor lo que se entiende por "modo". Así, los estudios de la gramática generativa atienden a rasgos semánticos y sintácticos como principios organizadores y aquellos de orientación pragmática se dirigen, por' antonomasia, a la relación (e intención) del enunciado lingüístico con los participantes en el acto comunicativo.

El deslinde, por lo menos desde una óptica metodológica, parece muy necesario en cuanto haría posible una mejor descripción de los aspectos involucrados. Por ello, creemos conveniente establecer ciertos criterios definitorios que den fundamento a cualquier análisis en este campo.

Definitivamente, el modo subjuntivo reúne, en español, diversos usos que parecieran no querer reducirse a una regla común o por lo menos bastante abarcadora. Cuando se trabaja, por ejemplo, con un conjunto de datos recolectados a partir de un corte sincrónico de los hechos de habla, no podemos olvidar que ellos traen consigo toda una carga evolutiva. El subjuntivo español no es el producto de una invención dada en un momento preciso de nuestra historia lingüística, sino más bien la suma de diversos usos que el sistema, en su evolución, fue acomodando para hacerse más efectivo. Por ejemplo, los usos del imperfecto de subjuntivo con valor de pretérito de indicativo (en el discurso periodístico, por ejemplo) sólo podrían explicarse desde una perspectiva diacrónica, en tanto esa forma en \{-ra\} proviene del primitivo pluscuamperfecto latino. El subjuntivo latino, como tal, no evolucionó directamente al español con sus mismos significados y usos. De manera que lo que encontramos en la lengua española actual agrupado bajo la etiqueta de subjuntivo, son usos diversos que en su momento no pertenecieron a esta categoría, pero que se incluyeron ahí por necesidades de comunicación y de reorganización interna del sistema.

Por otra parte, el mantenimiento de la nomenclatura gramatical latina para describir la lengua española ha obscurecido los hechos mencionados anteriormente, en vista de que referirse al modo subjuntivo como un conjunto heterogéneo de formas verbales que tienen un uso reducible a reglas específicas no corresponde exactamente con la realidad de los hechos.

Partiendo de estos supuestos, es necesario, como apuntábamos arriba, deslindar entre lo que sería el modo, como categoría gramatical, constituida por un conjunto de morfemas que afectan a las formas verbales y cuya derivación se puede rastrear desde el latín, de lo que sería la modalidad, como categoría básicamente pragmática (del análisis del discurso, si se quiere), que nos permitiría explicar el uso de aquellas formas que no se ajustan al conjunto, como por ejemplo, el uso del subjuntivo en sustitución del modo imperativo o aquellos casos en los que debería emplearse, de acuerdo con los principios gramaticales propuestos, el subjuntivo y en su lugar se usa el indicativo.

La noción de modalidad, por supuesto, no es nueva dentro de los estudios lingüísticos. De alguna forma Lenz, por ejemplo, trataba de apuntar hacia una caracterización de los modos gramaticales usando esta noción, del mismo modo que lo hacen todos aquellos análisis que siguen sus principios.

Mariner Bigorra (1971) había lanzado la inquietud de deslindar las nociones teóricas que sustentan la descripción de los modos en español, basándose principalmente en sus estudios del verbo latino. Para él, existe una clara contradicción entre los principios que se siguen 
para describir el modo, y por ello trata de aclararlos y, a la vez, describir el sistema verbal de acuerdo con su visión. Las ideas de Mariner, en efecto, aportan mucha luz al respecto, en tanto niegan que el concepto de subordinación sea suficiente para dar cuenta del uso del subjuntivo y que, más bien, la noción de modalidad se ajustaría mejor si pensamos que en la actitud del hablante explica aquellos casos en que ni el contexto semántico ni sintáctico funcionan. Piénsese, por ejemplo, en cláusulas del tipo Puede tomar sus vacaciones cuando usted quiera/quiere, en la que la diferencia modal sólo podría explicarse a partir de nociones actitudinales.

El aporte de Mariner es importante en cuanto que aclara parámetros gramaticales que se han usado híbridamente para la caracterización modal. Concretamente, encuentra que existe una triple noción con que se ha caracterizado al modo: como forma linguiística capaz de expresar la modalidad, como expresión de la actitud del hablante y como indicador de la subordinación. Este último, según su criterio y también de acuerdo con lo hasta ahora expuesto, no valdría como rasgo caracterizador y, por lo tanto, habría que valerse de los dos primeros para encontrar parámetros más definitorios.

Jiménez Juliá (1989), partiendo de estos criterios, los concreta aún más. Plantea que el modo verbal es diferente de la modalidad y el modus clausal: "Frente a la modalidad, que es un concepto preidiomático, o el modo verbal, que es una categoría gramatical, el conjunto de valores que incluimos aquí bajo la denominación de modus tiene un carácter semántico. El modus es el término con el que denominamos los juicios explícitos del hablante sobre su propio mensaje, que incluyen su certeza o incertidumbre, su deseo, etc., expresados por medios gramaticales" (199-200). En nuestro criterio, tratar de separar modus clausal de modalidad lleva a este lingüista a caer en terrenos un tanto peligrosos, pues se trata de separar lo que en su esencia está unido: los mecanismos gramaticales que usa el hablante para expresar el modus clausal redundan en el mismo efecto en el nivel de la modalidad. En su descripción, la modalidad queda fuera del sistema verbal, como una categoría preidiomática(?) que sería entonces de difícil ubicación dentro de la esfera de lo gramatical. En este caso, sería mejor mantener la separación básica entre modo (tal y como se ha definido) y modalidad, como la define Halliday (1970):

\footnotetext{
Modality is a form of participation by the speaker in the speech event. Through modality, the speakers associates with the thesis an indication of its status and validity in his own judgement; he intrudes, and takes up a position. Modality thus derives from what we called above the "interpersonal" function, language as a expression of role (335).
}

El mismo concepto de modalidad puede ser concretado si nos atenemos a lo que plantea Concepción Otáola (1988). Para ella, la modalidad habría que buscarla en dos planos: el de la enunciación y el del enunciado. En la primera, entran en juego hablante-oyente como base del proceso comunicativo y, de acuerdo con eso, tendríamos tres modalidades de la enunciación: declarativa, interrogativa e imperativa, como únicas formas de relación entre los participantes en el hecho de habla. Las modalidades del enunciado se corresponden con la noción de Halliday y con el modus clausal de Jiménez Juliá, en tanto expresan la relación del hablante frente a su enunciado. Esta lingüista distingue dos grupos básicos de modalidades del enunciado: las lógicas (manera en que el sujeto presenta el predicado como verdadero, contingente o probable) y las apreciativas (maneras en que el sujeto sitúa el enunciado con relación a juicios apreciativos). 
La modalidad, así definida, puede manifestarse por principios léxicos (adverbios de duda, por ejemplo, o verbos como querer, poder o deber ), por medio de categorías morfosintácticas (el modo subjuntivo sería sólo un medio de expresar ciertas modalidades) o inclusive fonéticos (la entonación de la frase).

Como se puede apreciar, el problema de la definición del modo subjuntivo radica esencialmente en que se ha partido de criterios no claros. La caracterización sintáctica que lo define como el modo de la subordinación no es privativa de este modo y, por ende, tampoco sería definitoria. Las taxonomías que se fundamentan en la actitud del hablante (el modus) confunden generalmente "modo" y "modalidad", olvidando que el subjuntivo es sólo un medio para expresar esta última. Por ello, si se quiere trabajar con el sistema verbal, hay que visualizarlo como eso, un sistema en el que las diferentes relaciones de tiempos y modos contribuyen a la expresión ya sea de la modalidad de la enunciación o la del enunciado, tal y como debería conceptualizarse en cualquier estudio que aborde el modo subjuntivo.

\section{Notas}

1. Debe aclararse que, quizás por las circunstancias, los estudios de la lingüística estadounidense tienden más a la teorización con fines pedagógicos o comprobativos de ciertos marcos de referencia, en tanto que dentro del mundo hispano se han orientado hacia el trabajo de campo, para lo cual la dialectología y la sociolingüística han aportado instrumentos básicos de trabajo para la caracterización de la lengua española.

\section{Bibliografía}

Alarcos Llorach, Emilio. 1994. "Estructura del verbo español." Estudios de gramática funcional del español. Madrid: Gredos, pp. 50 - 89.

1995. Gramática de la lengua española. Madrid: Espasa Calpe.

Bell, Anthony. 1990. "El modo en español: consideración de algunas propuestas recientes." Indicativo y Subjuntivo. Compilado por Ignacio Bosque. Madrid: Taurus Universitaria, pp. 81-106.

Bello, Andrés.1988. Gramática castellana. Estudio y edición de Ramón Trujillo.Madrid: Arco Libros S.A.

Bergen, John J. 1978. "One rule for Spanish subjunctive." Hispania. 61: 219 - 234.

Bosque, Ignacio. 1990. "Las bases gramaticales de la alternancia modal. Repaso y balance.". Indicativo y Subjuntivo. Compilado por Ignacio Bosque. Madrid: Taurus Universitaria, pp. 13-65. 
Castronovo, Brian J. 1990 "La categoría verbal de modo en la tradición gramatical española." Indicativo y Subjuntivo. Compilado por Ignacio Bosque. Madrid: Taurus Universitaria, pp. 66-80

Gili Gaya, Samuel. 1993. Curso Superior de sintaxis española. Barcelona: Vox.

Goldin, Mark. 1974. "A psychological perpsective of the Spanish Subjunctive." Hispania. 57: 295-300.

Guillaume, Gustave. 1929. Temps et verbe. Theorie des aspects, des modes et des temps. Paris: Honoré Champion.

Guitart, Jorge. 1990. “Aspectos pragmáticos del modo en los complementos de predicados de conocimiento y de adquisición de conocimiento en español." Indicativo y Subjuntivo. Compilado por Ignacio Bosque. Madrid: Taurus Universitaria, p.p. 315-329.

Hadlich, Roger L. 1982. Gramática transformativa del español. Madrid: Gredos.

Halliday, M.A.K. 1970. "Functional diversity in language as seen from a consideration of modality and mood in english." Foundations of Language. 6: 322-361.

Jiménez Julia, Tomás. 1989. "Modalidad, modo verbal y modus clausal en español." Verba: Anuario Galego de Filoloxia. 16: 175 - 214.

Keniston, Hayward. 1937. Spanish syntax list: A statistical study of grammatical usage. New York: Holt.

Klein, Flora. 1994. "The Painless Subjunctive." Studies in language learning and spanish linguistics. In honor Tracey Terrel. Editado por Hashemipour, P. Maldonado, R. y van Naerssen, M. New York: McGraw-Hill, Inc. Pp. 419-430.

Lamíquez, Vidal. 1982. El sistema verbal del español. Málaga: Librería Agora.

Lens, Rodolfo. 1920. La oración y sus partes. Madrid: Centro de Estudios Históricos.

Mariner Bigorra, S. 1971. "Triple noción básica en la categoría modal castellana." Revista de Filología Española. LIV, 3-4: 209 - 252.

Molho, Mauricio. 1975. Sistemática del verbo español (aspectos, modos y tiempos). Madrid: Gredos.

Navas Ruiz, Ricardo. 1990. "El subjuntivo castellano. Teoría y bibliografía crítica." Indicativo y Subjuntivo. Compilado por Ignacio Bosque. Madrid: Taurus Universitaria, pp. 107-141. 
Otáola Olano, Concepción. 1988. "La modalidad (con especial referencia a la lengua españo1a). Revista de Filología Española. 68. 1-2 : 97-117

Pottier, Bernard. 1970. Gramática del español. Madrid: Ediciones Alcalá, S.A.

Real Academia Española. 1973. Esbozo de una nueva gramática de la lengua española. Madrid: Espasa Calpe.

Spaulding, Robert. 1931. Syntax of the Spanish verb. England: Liverpool University Press.

Terrel, Tracey y Hooper, J. 1974. "A semantically based analysis of mood in Spanish." Hispania. 57: 484-494. 